\title{
Mapping spacetimes with LISA: inspiral of a test body in a 'quasi-Kerr' field
}

\author{
Kostas Glampedakis ${ }^{1}$ and Stanislav Babak ${ }^{2}$ \\ ${ }^{1}$ School of Mathematics, University of Southampton, Southampton SO17 1BJ, UK \\ ${ }^{2}$ Max Planck Institute (Albert Einstein Institute), Am Muehlenberg 1, D-14476 Golm, Germany
}

Received 25 October 2005

Published 1 June 2006

Online at stacks.iop.org/CQG/23/4167

\begin{abstract}
The future LISA detector will constitute the prime instrument for high-precision gravitational wave observations. Among other goals, LISA is expected to materialize a 'spacetime-mapping' program that is to provide information for the properties of spacetime in the vicinity of supermassive black holes which reside in the majority of galactic nuclei. Such black holes can capture stellarmass compact objects, which afterwards slowly inspiral under the emission of gravitational radiation. The small body's orbital motion and the associated waveform observed at infinity carry information about the spacetime metric of the massive black hole, and in principle it is possible to extract this information and experimentally identify (or not!) a Kerr black hole. In this paper we lay the foundations for a practical spacetime-mapping framework. Our work is based on the assumption that the massive body is not necessarily a Kerr black hole, and that the vacuum exterior spacetime is stationary axisymmetric, described by a metric which deviates slightly from the known Kerr metric. We first provide a simple recipe for building such a 'quasi-Kerr' metric by adding to the Kerr metric the leading order deviation which appears in the value of the spacetime's quadrupole moment. We then study geodesic motion of a test body in this metric, mainly focusing on equatorial orbits, but also providing equations describing generic orbits formulated by means of canonical perturbation theory techniques. We proceed by computing approximate 'kludge' gravitational waveforms which we compare with their Kerr counterparts. We find that a modest deviation from the Kerr metric is sufficient for producing a significant mismatch between the waveforms, provided we fix the orbital parameters. This result suggests that an attempt to use Kerr waveform templates for studying extreme mass ratio inspirals around a non-Kerr object might result in serious loss of signal-to-noise ratio and total number of detected events. The waveform comparisons also unveil a 'confusion' problem, that is the possibility of matching a true non-Kerr waveform with a Kerr template of different orbital parameters.
\end{abstract}

PACS numbers: 04.30.D6, 04.25.-g, 04.80.Cc 


\section{Introduction}

During the past several years astronomical observations have provided almost indisputable evidence in favour of the existence of 'dark' supermassive objects (with a mass spectrum $\sim 10^{5}-10^{9} M_{\odot}$ ) in the majority of galactic cores, including our own Milky Way [1]. Conventional wisdom dictates that these objects should be Kerr black holes as described by general relativity. This belief is (so far) rather based on our faith in general relativity itself than on hard 'experimental' evidence 3 . Other candidate massive objects have been proposed (such as soliton stars, boson stars, P-stars, gravastars and dark energy stars [3]) but these are treated with scepticism by the community as they involve 'exotic' physics.

The future LISA gravitational wave detector [4] is expected to be able to give a definitive answer to whether these massive objects are Kerr black holes or not. It is commonly accepted [5] that LISA will have the potential of 'mapping' the spacetime in these objects' close vicinity. This can be achieved by detection of gravitational radiation emitted during the inspiral of a stellar-mass compact body around the supermassive object. It is expected that LISA will be capable of detecting from several to about a thousand of these extreme mass ratio inspirals (EMRIs), during a 3-5 year mission [6]. For the actual detection and subsequent extraction of the system's physical properties, LISA's data analysis will rely heavily on matched filtering. This method is based on cross correlating the detector's noisy output with a pre-selected bank of waveform templates which should be accurate representations of the true signal. Among anticipated gravitational wave sources, EMRIs are thought of as one of the 'cleanest': they can be accurately modelled as a binary system of a 'test body' orbiting a Kerr black hole, evolving under its own gravitational dynamics, without receiving any other significant 'environmental' influence ${ }^{4}$.

The basic tool for studying such a system is black hole perturbation theory: the small body of mass $\mu \sim 1-10 M_{\odot}$ perturbing the spacetime of the much more massive black hole (of mass $M \sim 10^{6}$ ). For recent detailed reviews on EMRIs and further references on the subject we refer the reader to $[8,9]$.

In their great majority, EMRI-related studies take for granted the Kerr identity of the supermassive object. However, if we ever hope to materialize any spacetime-mapping program with LISA we should be prepared to dismiss (at least to some degree) this assumption. In fact, any claim that LISA will be able to probe the Kerr metric would require the use of realistic waveform templates which contain — in some suitable parametric form-deviations from the Kerr metric. The task of a LISA data analyst would be to quantify these deviations, most likely pointing to a null result.

This idea was first put forward by Fintan Ryan in the late 1990s. In a series of papers [10], Ryan demonstrated how LISA could in principle construct a map of the massive body's spacetime by means of EMRIs observations. His method is based on writing the general stationary axisymmetric vacuum metric in terms of multipole moments as prescribed in [11] (see also [12] for an extension to the electrovacuum case). These can be mass moments $M_{\ell}$ and current moments $S_{\ell}$, both labelled by the angular integer eigenvalue $\ell \geqslant 0$. Given the moments, the metric takes the symbolic form [10,11],

\footnotetext{
3 Note, however, that the existence of an event horizon seems to be required in order to explain the spectral properties of x-ray emission by galactic black hole binaries and AGNs, see for example [2].

4 This should be true for the majority of galactic supermassive black holes which are in a 'quiescent' accretion state. For the small fraction of strongly accreting black holes (like the ones in AGNs) the presence of an accretion disc may have non-negligible effects on a orbiting body (see for example [7]).
} 


$$
\begin{aligned}
g_{a b} \sim \sum_{n=0}^{\infty}\left[\frac{1}{r^{2 n+1}}\left\{M_{2 n} \mathcal{P}_{n}^{1}(\theta)+\left(M_{\ell<2 n}, S_{\ell<2 n}\right)\right\},\right. \\
\left.\frac{1}{r^{2 n+3}}\left\{S_{2 n+1} \mathcal{P}_{n}^{2}(\theta)+\left(M_{\ell<2 n+1}, S_{\ell<2 n+1}\right)\right\}\right],
\end{aligned}
$$

where $\mathcal{P}_{n}^{1,2}(\theta)$ are known angular functions and angular brackets stand for the contribution of lower moments (if they exist for given $n$ ).

These multipole moments will be clearly encoded in the geodesic equations of motion ${ }^{5}$ for a test body and consequently in the phase and amplitude of the emitted waveform. LISA (as any other gravitational wave detector) will be capable of tracking this phase with high precession and extract valuable information concerning the spacetime's multipolar structure.

The key feature that makes this method so attractive is that the Kerr spacetime is special, in the sense that all of its higher moments are 'locked' to the first two, the mass $M=M_{0}$ and spin $J=S_{1}[11]$,

$$
M_{\ell}+\mathrm{i} S_{\ell}=M(\mathrm{i} a)^{\ell},
$$

where $a=J / M$ is the familiar Kerr spin parameter. This relation is unique to Kerr holes and is a mathematical expression of the famous 'no-hair' theorem [13]. In practise this means that extracting just the three lowest moments, $M, J$ and the quadrupole moment $M_{2}$, could provide sufficient information for disproving that the central body is a Kerr black hole, assuming that (1.2) is not satisfied. The opposite case would strongly suggest a true Kerr black hole. Measurement of additional moments could pinpoint the identity of the body ${ }^{6}$. We should emphasize that a possible non-Kerr multipolar structure does not necessarily imply a non-Kerr identity for the massive object. A true Kerr hole with a substantial amount of material in its vicinity (say, in the form of an accretion disc) could effectively behave as a non-Kerr object. This scenario is not included in our present discussion but certainly merits a future detailed investigation.

Ryan's multipole expansion scheme is certainly an elegant and powerful method for 'mapping spacetimes', nevertheless there are some serious issues regarding its practicality. Computing gravitational waveforms for EMRIs requires (i) precise knowledge of a test body's geodesic motion and (ii) a wave-emission formalism that allows the calculation of waveforms and fluxes, once the orbital motion is prescribed. For the case of a Kerr black hole, geodesic motion is well known and extensively studied [16], while wave dynamics is studied with the help of the celebrated Teukolsky formalism [17] which encapsulates the dynamics of gravitational perturbations in one single master equation. However, it is not always appreciated that the study of both orbital and wave dynamics is greatly simplified due to the speciality of Kerr spacetime. In particular, one is able to first decouple and then separate the dynamical equations thanks to the Petrov type- $D$ character of the Kerr spacetime and a suitable choice of coordinate frame $[18,19]$.

Life is considerably more complicated if we consider a non-Kerr spacetime with arbitrary multipolar structure. To begin with, according to (1.1) an accurate representation of the metric in the strong field regime (the most relevant part of the inspiral for LISA) requires the inclusion of a large number of multipoles. This point can be made clearer if we note that the metric (1.1) is an expansion in $1 / r$ around the flat Minkowski spacetime. A more suitable expansion would be around (say) the Schwarzschild spacetime. We will return to this point in future work.

\footnotetext{
5 Multipolar expansion of the gravitational potential is a powerful technique in Newtonian gravity for performing geodesic measurements: the motion of a satellite around the Earth provides information on our planet's 'bumpiness'. 6 For example, a rotating massive Boson star [14] has three independent moments and its higher moments certainly do not obey relation (1.2).
} 
Complications first appear at the level of geodesic motion. The spacetime's stationaryaxisymmetric character guarantees the existence of two integrals of motion, the energy $E$ and the angular momentum (along the symmetry axis) $L_{z}$. However, the third integral of motion (the famous Carter constant for the Kerr metric) will typically be lost, as the Hamilton-Jacobi equation is not fully separable (see [13] for the solution of this equation in the Kerr case). Two integrals of motion are sufficient (together with initial conditions) for fully describing motion only for the special case of equatorial orbits. For generic orbits (which is the case relevant for LISA) the insufficient number of integrals of motion would force us to use the second-order geodesic equation of motion. This would result in a more complicated description of geodesic motion (as compared to the Kerr case), but technical complication is not the sole problem here. Typically, orbits with an insufficient number of integrals of motion exhibit chaotic dynamical behaviour which in turn would have a great impact on LISA data analysis.

The picture appears even more blurred when one attempts to study wave dynamics in a non-Kerr metric. Lack of decoupling for the perturbation equations inhibits the formulation of a 'Teukolsky-like' equation - the most versatile method in black hole perturbation theory is now lost. An alternative way of computing rigorous gravitational waveforms and fluxes would be the direct numerical time evolution of metric perturbations (after separation of the azimuthal $\phi$ coordinate) which come in a package of ten coupled partial differential equations (combined with four gauge-condition equations) [13].

Motivated (and partially discouraged!) by the above complications, we adopt a somewhat different (albeit less general) approach in the present work. Based on the belief that the massive objects in galactic nuclei are most likely Kerr black holes, we only address the question: is the spacetime around these objects described by the exact Kerr metric, or by a slightly different metric? We do not attempt to identify the source of this metric, in fact we are agnostic on this issue. Our less ambitious goal is to quantify the deviation from the Kerr metric.

This point should be emphasized because most of the other candidate objects cannot be considered in any sense as being 'almost' a Kerr black hole. The idea of building a 'quasiblack hole' spacetime has been recently advocated by Collins and Hughes [20]. These authors constructed a 'bumpy' Schwarzschild black hole by adding a certain amount of quadrupole moment (in the form of a given mass distribution outside the black hole) and then studied equatorial orbits in the resulting spacetime.

In our scheme we modify the quadrupole moment of the exact Kerr metric (this is the lowest moment where Kerr can be distinguished from the spacetime of another axisymmetric/stationary body). This procedure is performed in a natural way with the help of the well-known Hartle-Thorne exterior metric [22]. The resulting 'quasi-Kerr' metric is customized for use in strong gravity situations and has the nice feature of smoothly reducing to the familiar Kerr metric in Boyer-Lindquist (BL) coordinates. Being arbitrarily close to the BL Kerr metric (which allows full separability of the Hamilton-Jacoby and wave equations) allows us to formulate first-order equations of motion and integrals of motion by perturbing the well-known Kerr equations. Since the number of integrals of motion is conserved in this approximation, the resulting motion is guaranteed to be integrable. As yet, we have not explored whether the same trick would lead to a 'quasi-Teukolsky' perturbation equation. For the purposes of the present investigation it is quite sufficient to use the so-called hybrid approximation [23], [24] and compute 'kludge' waveforms. Lastly, we need to emphasize that our quasi-Kerr metric is an approximate vacuum solution, and consequently not suitable for describing a Kerr black hole with a distribution of matter in its vicinity.

The paper is structured as follows. In section 2 we provide the prescription for building our quasi-Kerr metric. This is the paper's main result. Section 3 is devoted to the solution of the Hamilton-Jacobi equation in the quasi-Kerr metric. We first discuss the issue of 
separation of variables for this equation and then proceed with the derivation of equations of motion based on standard canonical perturbation theory. Section 3.3 is dedicated to the study of equatorial orbits and comparison of orbital frequencies and periastron advance for Kerr and quasi-Kerr orbits. In section 4 we compute approximate waveforms from test bodies in equatorial orbits of the quasi-Kerr metric. These are compared to their Kerr analogues by calculating their overlap function in the LISA sensitivity band. In the same section we touch upon the problem of 'confusion' between Kerr and quasi-Kerr waveforms. A summary and concluding discussion can be found in section 5. Appendices containing some technical details, including a review of the action-angle formalism in the Kerr spacetime can be found at the end of the paper. Throughout the paper we use geometrized units $G=c=1$ and adopt a $\{-,+,+,+\}$ signature for the metric. We repeatedly use the labels ' $\mathrm{K}$ ' and 'qK' for 'Kerr' and 'quasi-Kerr', respectively.

\section{Building a quasi-Kerr spacetime}

As we mentioned in the introduction, the central idea of our work is to replace the general multipolar expansion (1.1) of an axisymmetric-stationary vacuum spacetime with a less general 'quasi-Kerr' metric. In the language of multipole moments this means

$$
\begin{aligned}
& M_{\ell}=M_{\ell}^{\mathrm{K}}+\delta M_{\ell}, \quad \ell \geqslant 2 \\
& S_{\ell}=S_{\ell}^{\mathrm{K}}+\delta S_{\ell}, \quad \ell \geqslant 3,
\end{aligned}
$$

where $\delta M_{\ell}, \delta S_{\ell}$ are assumed small. With respect to the first two leading moments $M, J$ the spacetimes outside a Kerr hole and any other stationary axisymmetric rotating body are indistinguishable. Essentially, $M$ and $J$ can take the same value for both bodies (the spin is presumably limited, for a Kerr hole $a \leqslant M$ ). This degeneracy is broken as soon as we take into account the next most important moment, the mass quadrupole $M_{2}$. In our scheme we choose to take into account only the deviation in the quadrupole moment $\mathcal{Q} \equiv M_{2}$ and neglect any deviation in all higher moments. We introduce the dimensionless deviation parameter $\epsilon$ as

$$
\mathcal{Q}=\mathcal{Q}^{\mathrm{K}}-\epsilon M^{3}, \quad \text { where } \quad \mathcal{Q}^{\mathrm{K}}=-\frac{J^{2}}{M} .
$$

Our quasi-Kerr metric takes the form

$$
g_{\alpha \beta}=g_{\alpha \beta}^{\mathrm{K}}+\epsilon h_{\alpha \beta}+\mathcal{O}\left(\delta M_{\ell \geqslant 4}, \delta S_{\ell \geqslant 3}\right),
$$

where $g_{\alpha \beta}^{\mathrm{K}}$ is the exact Kerr metric. Our goal is to determine the metric functions $h_{\alpha \beta}(r, \theta)$.

This can be achieved by using the Hartle-Thorne (HT) metric [22] which describes the vacuum spacetime outside any slowly rotating axisymmetric and stationary body. Indeed, this metric is fully accurate up to the quadrupole moment (which scales quadratically with the body's spin), and it includes as a special case the Kerr metric (at $\mathcal{O}\left(a^{2}\right)$ order). It is then possible to isolate the leading-order quadrupole moment deviation and deduce the $h_{\alpha \beta}$ piece in (2.4). Implicit in this prescription is the assumption that the massive body's exterior spacetime has nonzero higher moments induced by the body's rotation, in the same sense that a black hole has nonzero moments (with $\ell>0$ ) only when $a \neq 0$. As a consequence, terms of order $\mathcal{O}\left(\epsilon a, \epsilon^{2}\right)$ are beyond the desired accuracy and therefore neglected. Unlike the general multipole expansion (1.1), our prescription by construction offers no information (regarding 
possible deviations from the Kerr values) on moments higher than the quadrupole ${ }^{7}$. On the other hand our metric is not an expansion in inverse powers of $r$, instead is written in a compact fully relativistic form suitable for strong-field conditions.

We move on to the calculation of $h_{a b}$. For our purposes we shall only need the exterior HT metric [22] which is expressed in terms of the dimensionless parameters,

$$
j \equiv \frac{J}{M^{2}}, \quad q \equiv-\frac{\mathcal{Q}}{M^{3}} .
$$

Then at $\mathcal{O}\left(j^{2}\right)$ accuracy, the metric is given by (these expressions are taken from [25] after correcting a typographical sign error in the $F_{1}$ function),

$$
\begin{aligned}
& g_{t t}^{\mathrm{HT}}=-(1-2 M / r)\left[1+j^{2} F_{1}+q F_{2}\right] \\
& g_{r r}^{\mathrm{HT}}=(1-2 M / r)^{-1}\left[1+j^{2} G_{1}-q F_{2}\right] \\
& g_{\theta \theta}^{\mathrm{HT}}=r^{2}\left[1+j^{2} H_{1}+q H_{2}\right] \\
& g_{\phi \phi}^{\mathrm{HT}}=r^{2} \sin ^{2} \theta\left[1+j^{2} H_{1}+q H_{2}\right] \\
& g_{t \phi}^{\mathrm{HT}}=-\frac{2 M^{2} j}{r} \sin ^{2} \theta .
\end{aligned}
$$

The functions $F_{1,2}(r, \theta), H_{1,2}(r, \theta)$ appearing in these expressions are given in appendix A. Note that the metric (2.6) is written in the coordinate frame $\{t, r, \theta, \phi\}$ originally used by Hartle and Thorne. Without loss of generality, we can write

$$
q=j^{2}+\epsilon
$$

Clearly, the limit $\epsilon \rightarrow 0$ corresponds to the HT representation of the Kerr spacetime ${ }^{8}$.

For reasons we discuss later in the paper, it is highly desirable for the quasi-Kerr metric to reduce to the familiar Kerr metric in Boyer-Lindquist (BL) coordinates in the limit $\epsilon \rightarrow 0$. The transformation between BL and the original HT coordinates was provided by Hartle and Thorne ${ }^{9}$ :

$$
\begin{aligned}
t_{\mathrm{BL}} & =t, \quad \phi_{\mathrm{BL}}=\phi \\
r_{\mathrm{BL}} & =r-\frac{j^{2} M^{2}}{2 r^{3}}\left[(r+2 M)(r-M)-\cos ^{2} \theta(r-2 M)(r+3 M)\right] \\
& \equiv r-j^{2} M^{2} f_{\mathrm{BL}}(r, \theta) \\
\theta_{\mathrm{BL}} & =\theta-\frac{j^{2} M^{2}}{2 r^{3}}(r+2 M) \sin \theta \cos \theta \equiv \theta-j^{2} M^{2} g_{\mathrm{BL}}(r, \theta) .
\end{aligned}
$$

Written in this manner, this transformation is not really practical for someone wishing to obtain the metric in BL coordinates. Fortunately, inverting the above equations with respect to the

7 It should be pointed out that the approximation of neglecting $\delta M_{\ell}, \delta S_{\ell}$ beyond the quadrupole order does not necessarily imply that these moments are not important. However, it appears natural to expect that the gravitational field of a generic non-Kerr massive object would more prominently deviate from the Kerr field at the first possible level, i.e. the quadrupole moment, provided that rotation is not extreme. Hence it makes sense to include only this contribution in an initial attempt to model non-Kerr fields.

8 To convey an idea on the range of values that $\epsilon$ could take, we use the results of Laarakkers and Poisson [21] for the quadrupole moment of rotating neutron stars. They show that the quadrupole moment is well approximated as $q \approx b j^{2}$. The factor $b$ is a function of the mass and the equation of state, with a value varying between $\sim 2-12$ (decreasing with mass and increasing with stiffness of the equation of state). It was emphasized in [21] that this relationship holds even for fast rotations. The fact that $b$ is positive reflects oblateness of the mass distribution. Using this relationship we obtain $\epsilon=(b-1) j^{2}$, which gives an average value $\epsilon \sim 5 j^{2}$, for a star with mass $M \sim 1 M_{\odot}$.

9 Note that both the original transformation equations by Hartle and Thorne and their recent reproduction in [25] contain a sign error (see also [26]). 
HT coordinates is a trivial task. According to (2.10) $r_{\mathrm{BL}}=r+\mathcal{O}\left(j^{2}\right)$ and $\theta_{\mathrm{BL}}=\theta+\mathcal{O}\left(j^{2}\right)$; therefore we are free to use either set of coordinates in the functions $f_{\mathrm{BL}}, g_{\mathrm{BL}}$ since these appear in the $\mathcal{O}\left(j^{2}\right)$ terms. Thus, the inverse transformation is simply,

$$
r=r_{\mathrm{BL}}+j^{2} M^{2} f_{\mathrm{BL}}\left(r_{\mathrm{BL}}, \theta_{\mathrm{BL}}\right) \quad \theta=\theta_{\mathrm{BL}}+j^{2} M^{2} g_{\mathrm{BL}}\left(r_{\mathrm{BL}}, \theta_{\mathrm{BL}}\right) .
$$

Hereafter, we shall drop the subscript on the BL coordinates. As the two sets of coordinates differ at order $\mathcal{O}\left(j^{2}\right)$ the quadrupole-order pieces in (2.6) are immune with respect to the transformation (2.11). Only the 'Schwarzschild' portion of the metric is affected by the coordinate transformation. For the contravariant metric components we obtain

$$
\begin{aligned}
& g_{\mathrm{HT}}^{t t}=g_{\mathrm{Ka}^{2}}^{t t}+\epsilon(1-2 M / r)^{-1}\left[f_{3}(r)+f_{4}(r) \cos ^{2} \theta\right] \\
& g_{\mathrm{HT}}^{r r}=g_{\mathrm{Ka}^{2}}^{r r}+\epsilon(1-2 M / r)\left[f_{3}(r)+f_{4}(r) \cos ^{2} \theta\right] \\
& g_{\mathrm{HT}}^{\theta \theta}=g_{\mathrm{Ka}^{2}}^{\theta \theta}-\frac{\epsilon}{r^{2}}\left[h_{3}(r)+h_{4}(r) \cos ^{2} \theta\right] \\
& g_{\mathrm{HT}}^{\phi \phi}=g_{\mathrm{Ka}^{2}}^{\phi \phi}-\frac{\epsilon}{r^{2} \sin ^{2} \theta}\left[h_{3}(r)+h_{4}(r) \cos ^{2} \theta\right] \\
& g_{H T}^{t \phi}=g_{\mathrm{Ka}^{2}}^{t \phi},
\end{aligned}
$$

where the functions $f_{3}, f_{4}, h_{3}, h_{4}$ are given in appendix A. In equations (2.12) we have denoted as $g_{\mathrm{Ka}^{2}}^{\alpha \beta}$ the $\mathcal{O}\left(a^{2}\right)$ Kerr metric (in BL coordinates) with $a=j M$. From equations (2.12) one can extract the desired deviation $h_{\alpha \beta}$.

Putting all the pieces together, the ansatz for building a quasi-Kerr metric in BoyerLindquist coordinates is

$$
g_{a b}=g_{a b}^{\mathrm{K}}+\epsilon h_{a b}
$$

where

$h^{t t}=(1-2 M / r)^{-1}\left[\left(1-3 \cos ^{2} \theta\right) \mathcal{F}_{1}(r)\right], \quad h^{r r}=(1-2 M / r)\left[\left(1-3 \cos ^{2} \theta\right) \mathcal{F}_{1}(r)\right]$

$h^{\theta \theta}=-\frac{1}{r^{2}}\left[\left(1-3 \cos ^{2} \theta\right) \mathcal{F}_{2}(r)\right], \quad h^{\phi \phi}=-\frac{1}{r^{2} \sin ^{2} \theta}\left[\left(1-3 \cos ^{2} \theta\right) \mathcal{F}_{2}(r)\right]$

$h^{t \phi}=0$.

The functions $\mathcal{F}_{1,2}(r)$ are given in appendix A.

Looking at the explicit form of $h_{\alpha \beta}$ it is evident that these functions are divergent at $r \rightarrow 2 M$. This would correspond to the location of the event horizon at the accuracy $\mathcal{O}\left(j^{2}\right)$ of the HT metric. Hence, there is no real mystery behind this behaviour which is also present in the case of the full Kerr metric. However, since in our scheme we do not associate the quasi-Kerr metric with a black hole we are forced to be agnostic regarding the nature of the 'surface' of the massive body. Whether it is an event horizon or something else is an issue we simply do not address. This lack of information has no significant impact on the study of the orbital motion of a test body, as long as we do not consider orbits too close to $2 M$ (assuming they exist).

The situation is not so clear when one tries to study wave dynamics in a quasi-Kerr metric and compute (say) fluxes. To begin with, we should mention that a first approximation would be to completely neglect any gravitational flux impinging on the 'surface', assuming that it is small compared to the fluxes at infinity. This is certainly true for the case of black holes $[27,28]$ and it is not unreasonable to expect the same in other scenarios. The main issue regards the computation of the fluxes at infinity, and their sensitivity to the absence of a 
concrete boundary condition at $r \sim 2 M$. One could try different boundary conditions, say, perfect reflection or free propagation and assess how robust the flux results are (see for example [10]). Other aspects of wave dynamics in a quasi-Kerr field, such as the existence and properties of quasi-normal modes, are extremely sensitive to boundary conditions and cannot be studied within our framework.

\section{Geodesic motion in the quasi-Kerr metric}

\subsection{The Hamilton-Jacobi equation: issues of separability}

An elegant method for deriving equations of motion for a point particle in a given gravitational potential is the Hamilton-Jacobi (HJ) formalism (see [29] for background material and appendix B of the present paper). The end product is the known $\mathrm{HJ}$ equation for the generating function $S$, which in its general relativistic version is written as

$$
\frac{1}{2} g^{\alpha \beta} \frac{\partial S}{\partial x^{\alpha}} \frac{\partial S}{\partial x^{\beta}}+\frac{\partial S}{\partial \lambda}=0
$$

where $\lambda$ is the geodesic affine parameter. In essence, this equation follows from the definition of the Hamiltonian for point-particle motion,

$$
H\left(x^{\alpha}, p_{\beta}\right)=\frac{1}{2} g^{\mu v} p_{\mu} p_{v}=-\frac{1}{2} \mu^{2}
$$

by replacing the four momenta $p^{\alpha}=\mathrm{d} x^{\alpha} / \mathrm{d} \lambda$ by $p^{\alpha}=g^{\alpha \beta} \partial S / \partial x^{\beta}$. Here the BL coordinates $x^{\alpha}=\{t, r, \theta, \phi\}$ are conjugate to $p^{\alpha}$.

One of the 'miracles' of the Kerr metric is that it allows full separability of the HJ equation, leading to the well-known Kerr geodesic equations of motion [13, 16]. Separability with respect to $\lambda, t, \phi$ generates the conserved quantities $\mu$ (the test body's mass), $E$ (energy) and $L_{z}$ (angular momentum along the symmetry axis). This is always possible as long as the metric is stationary axisymmetric. The special property of the Kerr metric (originating from its Petrov-type- $D$ character) is that it allows the additional, non-trivial, separability with respect to $(r, \theta)$, leading to the third constant $Q$, the Carter constant. This is possible only in a restricted family of coordinate frames, the BL one among them [18]. In fact, if we expand the Kerr metric with respect to $a$, then the HJ equation is separable at each individual order.

On the other hand, the HJ equation is not fully separable with respect to the HT metric (2.6), not even when the Kerr limit $\epsilon \rightarrow 0$ is taken. This reveals that the coordinate frame used in the original HT metric is not 'privileged' and consequently a bad choice for a quasiKerr metric. Instead, BL coordinates seem like a good choice as in the limit $\epsilon \rightarrow 0$ they admit separability. A similar conclusion can be reached for the general metric (1.1). In its present form it does not allow separation of the HJ equation (or of the scalar and gravitational perturbation equations) even when the Kerr limit is taken (which corresponds to enforcing relation (1.2)).

The HJ equation for the full quasi-Kerr metric (2.4) is solved by assuming the standard form

$$
S=\frac{1}{2} \mu^{2} \lambda-E t+L_{z} \phi+\mathcal{S}_{r}(r)+\mathcal{S}_{\theta}(\theta)
$$

with an extra expansion

$$
\mathcal{S}_{r, \theta}=S_{r, \theta}+\epsilon \hat{S}_{r, \theta},
$$

where $S_{r, \theta}$ solves the exact Kerr HJ equation. After separating out the Kerr portion of (3.1), and neglecting $\mathcal{O}\left(\epsilon a, \epsilon^{2}\right)$ terms, we are left with

$r(r-2 M)\left[2 \frac{\mathrm{d} S_{r}}{\mathrm{~d} r} \frac{\mathrm{d} \hat{S}_{r}}{\mathrm{~d} r}+f_{3}\left(\frac{\mathrm{d} S_{r}}{\mathrm{~d} r}\right)^{2}\right]+\frac{E^{2} r^{3} f_{3}}{r-2 M}-K h_{3}=-2 \frac{\mathrm{d} S_{\theta}}{\mathrm{d} \theta} \frac{\mathrm{d} \hat{S}_{\theta}}{\mathrm{d} \theta}+Z(r) \cos ^{2} \theta$, 
where $K$ is a separation constant and

$$
Z(r)=K h_{4}-f_{4} \frac{E^{2} r^{3}}{r-2 M}-f_{4} r(r-2 M)\left(\frac{\mathrm{d} S}{\mathrm{~d} r}\right)^{2} .
$$

This non-vanishing function spoils the separability of (3.5). In retrospect, this is not really surprising as the addition of the $\epsilon$-pieces 'contaminates' the Kerr metric in the sense that the resulting quasi-Kerr spacetime is not type- $D$ anymore, although (in some loose sense) it is arbitrarily 'close' (this is also the explanation for why the non-type- $D$ HT metric does not lead to a separable HJ equation).

At this point it would seem that no real advantage has being gained by using the quasi-Kerr metric (2.4) as the HJ equation is still non-separable with respect to $(r, \theta)$. However, the fact that the equation is separable in the $\epsilon \rightarrow 0$ limit can be exploited and by means of canonical perturbation theory [29] can lead to the desired equations of motion (see section 3.2).

Studying geodesic motion in a quasi-Kerr field is just the first step towards solving the EMRI problem. For gravitational wave observations it is pivotal to be able to compute accurate waveforms and fluxes. In the case of Kerr black holes, this is achieved by means of the Teukolsky equation [17] (see [8,9] for reviews relevant to EMRI, and further references), which governs the dynamics of perturbations of the Weyl scalars $\left\{\psi_{0}, \psi_{4}\right\}$ in the framework of the classic Newman-Penrose formalism. The remarkable result that all these perturbations are described by one single master equation is a consequence (once more) of the Petrov-type- $D$ character of the Kerr metric, which allows decoupling of the various perturbation equations [18]. Moreover, for a certain class of coordinate frames (the BL the most widely used one) the Teukolsky equation admits separation of variables, reducing the problem to the solution of simple ODEs. None of these properties survive for a non-type- $D$ metric such as (1.1) or (2.4). However, as the quasi-Kerr metric is 'almost' type- $D$ there may still be a chance of decoupling (at least partially) the perturbation equations for the Weyl scalars. This crucial issue is left for future work. In the unfortunate case that this expectation proves untrue, we may have to work with straight metric perturbations which are governed by ten coupled PDEs plus gauge conditions. These equations will still admit separation with respect to $\phi$ so they can be studied in the time domain in a ' $2+1$ ' format. In the present paper, the issue of wave dynamics will be dealt with by simply computing approximate waveforms ${ }^{10}$ (section 4 ), but our conclusions should be valid even if we had rigorous waveforms at our disposal.

\subsection{Generic orbits}

Although we are mainly concerned with equatorial orbits in this paper, we nevertheless derive approximate equations of motion for generic quasi-Kerr orbits. Our objective is to demonstrate how one can arrive at such equations despite the non-separability of the HJ equation and without resorting to the full second-order equations of geodesic motion. This subsection is somewhat detached from the paper's main guideline and the reader should first consult appendix B (where we review the action-angle formalism in the Kerr spacetime, following [30]) for definitions and notation used here.

Our calculation consists of nothing more than the application of canonical perturbation theory (see [29] for further details) to point-particle motion in the Kerr spacetime. The full Hamiltonian can be written as the sum of the Kerr point-particle Hamiltonian amended with the quadrupolar perturbation,

$$
H=\frac{1}{2} g_{K}^{\alpha \beta} p_{\alpha} p_{\beta}+\frac{1}{2} \epsilon h^{\alpha \beta} p_{\alpha} p_{\beta} \equiv H_{\circ}+\epsilon H_{1} .
$$

${ }^{10}$ A similar path was taken by Ryan who only calculated slow-motion, 'restricted post-Newtonian', waveforms from circular equatorial orbits [10]. 
The unperturbed Hamiltonian is a function of the actions only, $H_{\circ}=H_{\circ}\left(I_{\beta}\right)$, while the perturbation depends on both actions and angles, $H_{1}=H_{1}\left(w^{\alpha}, I_{\beta}\right)$. Expressing the Hamiltonian in this functional form is possible provided we have first obtained relations $x^{\kappa}\left(w^{\alpha}, I_{\beta}\right)$ and $p_{\kappa}\left(w^{\alpha}, I_{\beta}\right)$. For the Kerr problem, these are given by implicit relations, see appendix B.

In the unperturbed problem $H_{\circ}$ the actions are constants and the angles vary linearly with respect to $\lambda$. This is no longer true for the full perturbed Hamiltonian $H$. We seek a canonical transformation $\left\{w^{\alpha}, I_{\beta}\right\} \Rightarrow\left\{\hat{w}^{\alpha}, \hat{I}_{\beta}\right\}$ to a new set of angle actions which would have exactly the above properties. Clearly, the old and new variables will differ at $\mathcal{O}(\epsilon)$. The generating function takes the form [29],

$$
F\left(w^{\alpha}, \hat{I}_{\beta}\right)=w^{k} \hat{I}_{k}+\Phi\left(w^{\alpha}, \hat{I}_{\beta}\right) .
$$

Then we have

$$
I_{\alpha}=\frac{\partial F}{\partial w^{\alpha}}=\hat{I}_{\alpha}+\frac{\partial \Phi}{\partial w^{\alpha}} \quad \text { and } \quad \hat{w}^{\alpha}=\frac{\partial F}{\partial \hat{I}_{\alpha}}=w^{\alpha}+\frac{\partial \Phi}{\partial \hat{I}_{\alpha}} .
$$

Denoting as $K\left(\hat{I}_{\beta}, \epsilon\right)$ the new Hamiltonian we also have

$$
\begin{aligned}
& \frac{\mathrm{d} \hat{w}^{\alpha}}{\mathrm{d} \lambda}=\frac{\partial K}{\partial \hat{I}_{\alpha}} \Rightarrow \hat{w}^{\alpha}=\hat{v}_{\alpha} \lambda+\beta^{\alpha} \\
& \frac{\mathrm{d} \hat{I}_{\alpha}}{\mathrm{d} \lambda}=-\frac{\partial K}{\partial \hat{w}^{\alpha}}=0+\mathcal{O}\left(\epsilon^{2}\right),
\end{aligned}
$$

where $\hat{v}_{\alpha}$ are the new fundamental frequencies. The function $F$ solves the following HJ equation:

$$
H\left(w_{\alpha}, \frac{\partial F}{\partial w^{\alpha}}\right)=K\left(\hat{I}_{\beta}, \epsilon\right) .
$$

The standard choice for the Hamiltonian $K$ is

$$
K\left(\hat{I}_{\beta}, \epsilon\right)=H_{\circ}\left(\hat{I}_{\beta}\right)+\epsilon\left\langle H_{1}\right\rangle\left(\hat{I}_{\beta}\right),
$$

where we have defined the averaged perturbation with respect to the original angle variables,

$$
\left\langle H_{1}\right\rangle\left(\hat{I}_{\beta}\right) \equiv \oint \mathrm{d} w^{\alpha} H_{1}\left(w^{k}, \hat{I}_{\beta}\right) .
$$

Expanding the left-hand side of (3.12) we then find for the generating function

$$
v_{\alpha} \frac{\partial \Phi}{\partial w^{\alpha}}=\epsilon\left[\left\langle H_{1}\right\rangle-H_{1}\right]
$$

The perturbed equations of motion are derived by first inverting equations (3.9) with respect to the old action angles, isolating the secular changes in these relations and then substituting into the unperturbed equations $w^{\alpha}\left(x^{k}, I_{\beta}\right)$ (see equations (B.5) in appendix B). Note that in the function $\Phi$ we are free to interchange new with old actions at $\mathcal{O}(\epsilon)$ accuracy. From (3.15) and the fact that $\Phi\left(w^{\alpha}, I_{\beta}\right)$ is a periodic function of the angles $w^{\alpha}$ [29] we find that

$$
\left\langle\frac{\partial \Phi}{\partial w^{\alpha}}\right\rangle=0
$$

which implies that there are no secular changes in the actions at $\mathcal{O}(\epsilon)$. This also means that there is no secular change in $\left\{E, L_{z}, Q\right\}$. At leading order, quasi-Kerr orbits admit three integrals of motion, like in the Kerr geodesic motion. 
Averaging the perturbation in the angle variables $\left\langle\partial \Phi / \partial I_{\alpha}\left(w^{k}, I_{\beta}\right)\right\rangle=\left\langle\partial \Phi / \partial I_{\alpha}\right\rangle\left(I_{\beta}\right) \neq 0$ implies that in a secular sense $w^{\alpha}=\hat{v} \lambda+\hat{\beta}^{\alpha}$ where $\hat{\beta}^{\alpha}$ is a constant phase.

Therefore we have shown that the quasi-Kerr geodesic equations of motion can be directly derived from the corresponding Kerr equations (B.5) with the substitution $v_{\alpha} \Rightarrow \hat{v}_{\alpha}$ in the left-hand sides only. Despite the identical shapes between the Kerr and quasi-Kerr orbits, the difference in the frequencies will translate in a difference in precession rates for the periastron and for the orbital plane ${ }^{11}$.

Generic quasi-Kerr orbits will be discussed in detail in a future paper. Here we have shown that in a secular sense the motion has three conserved quantities (apart from $\mu$ ), which is an important property and stands above of what one would expect for a general non-Kerr axisymmetric-stationary metric like (1.1). As we mentioned in the introduction, geodesic motion in such metric is not formally integrable and may well lead to chaotic behaviour in the motion of a test body.

In fact, orbital dynamics in general axisymmetric-stationary gravitational fields are typically much different from the ones in spherically-symmetric fields or even the Kerr field. We can easily demonstrate this difference by asking the simple question: which axisymmetricstationary gravitational fields can support circular orbits? In Newtonian theory the answer is that only a special family of potentials with the form $V(r, \theta)=V_{\circ}(r)+V_{1}(\theta) / r^{2}$ (where $V_{\circ}, V_{1}$ arbitrary functions) admit orbits with $u^{r}=\mathrm{d} u^{r} / \mathrm{d} t=0$. In general relativity the same requirement for a given metric $g_{a b}(r, \theta)$ results in conditions involving the metric and its first derivatives. Once more, the Kerr metric is special as it satisfies these conditions. Further analysis of this issue will be presented in a future paper.

\subsection{Equatorial orbits}

For the special case of equatorial orbits the quasi-Kerr HJ equation (3.1) is trivially separable. The resulting equations of motion are

$$
\begin{aligned}
\left(u^{r}\right)^{2}=\left(\frac{\mathrm{d} r}{\mathrm{~d} \lambda}\right)^{2} & =V_{r}(r)=\left(E^{2}-1\right)+\frac{2 M}{r}-\left[L_{z}^{2}+a^{2}\left(1-E^{2}\right)\right] \frac{1}{r^{2}}+\frac{2 M}{r^{3}}\left(L_{z}-a E\right)^{2} \\
& -\epsilon\left(1-\frac{2 M}{r}\right)\left[\left(f_{3}-h_{3}\right) \frac{L_{z}^{2}}{r^{2}}+f_{3}\right] \\
u^{\phi}=\frac{\mathrm{d} \phi}{\mathrm{d} \lambda}=V_{\phi} & =\frac{1}{\Delta}\left[\frac{2 M}{r}\left(a E-L_{z}\right)+L_{z}\right]-\epsilon \frac{h_{3}}{r^{2}} L_{z} \\
u^{t}=\frac{\mathrm{d} t}{\mathrm{~d} \lambda}=V_{t} & =\frac{1}{\Delta}\left[E\left(r^{2}+a^{2}\right)+\frac{2 M a}{r}\left(E a-L_{z}\right)\right]-\epsilon\left(1-\frac{2 M}{r}\right)^{-1} f_{3} E
\end{aligned}
$$

In these equations we have normalized energy and angular momentum with the body's mass. In the standard manner, we express the test body's radial location in terms of a pair of orbital elements, the semi-latus rectum $p$ and the eccentricity $e$,

$$
r=\frac{p}{1+e \cos \chi} \text {. }
$$

\footnotetext{
${ }^{11} \mathrm{~A}$ basic feature of generic Kerr orbits is their characterization by three incommensurate orbital frequencies $\Omega_{i}=v_{i} / v_{t}$ (in BL time). This property results in orbits which are not closed, but rather exhibit precessional motions. Periastron precession is a consequence of $\Omega_{r} \neq \Omega_{\phi}$ and orbital plane precession (Lense-Thirring precession) is a consequence of $\Omega_{\theta} \neq \Omega_{\phi}$. Keplerian orbits, on the other hand, are fixed closed ellipses since their frequencies are degenerate, $\Omega_{r}=\Omega_{\theta}=\Omega_{\phi}$.
} 
In the weak-field limit $(p \gg M)$ these parameters coincide with the familiar Keplerian parameters. The parameter $\chi$ varies monotonically, while $r$ 'runs' between the two radial turning points, the periastron $r_{p}=p /(1+e)$ and the apastron $r_{a}=p /(1-e)$. The elements $(p, e)$ can be written as functions of $E, L_{z}$ (and vice versa) using $V_{r}\left(r_{a}\right)=V_{r}\left(r_{p}\right)=0$. For the Kerr orbits $V_{r}(r)$ is a cubic polynomial and the third root $r_{3}$ (which is the smallest of the three) can be used to define a 'separatrix' of bound/unbound orbits. The transition occurs when $r_{p}=r_{3}$ which defines an innermost stable bound orbit. In the quasi-Kerr case, the equation $V_{r}=0$ is more complicated, but since the equations of motion (3.19) deviate only slightly from the corresponding Kerr equations, we expect the quasi-Kerr separatrix to be located close to the Kerr separatrix [27] for the same $\{a, p, e\}$.

Equatorial orbits are characterized by a pair of orbital frequencies $\Omega_{r}, \Omega_{\phi}$. These are defined in terms of the radial period $T_{r}$ (the coordinate time required for the body to move from $r_{p}$ to $r_{a}$ and back), and of the periastron shift $\Delta \phi$ (total accumulated angle $\phi$ over a period $\left.T_{r}\right)$,

$$
\Omega_{r}=\frac{2 \pi}{T_{r}}, \quad \Omega_{\phi}=\frac{\Delta \phi}{T_{r}} .
$$

These frequencies are 'observables' as the emitted gravitational waveform (on an adiabatic timescale where backreaction is not significant) comes in discrete frequencies,

$$
\omega_{m k}=m \Omega_{\phi}+k \Omega_{r},
$$

where $k, m$ are integers (the latter associated with the azimuthal angle $\phi$ ). The appearance of a spectrum of the form (3.22) is a direct consequence of the periodicity of the motion. Note that in the Keplerian limit $\left(p \gg M\right.$ ) we have $\Delta \phi \rightarrow 2 \pi$, hence $\Omega_{r} \rightarrow \Omega_{\phi}$.

As a first stab at the problem of comparing Kerr versus quasi-Kerr EMRIs we calculate the above frequencies $\Omega_{r}, \Omega_{\phi}$ for identical values $\{a, p, e\}$ while varying the quadrupole deviation $\epsilon$. We use the simple expressions,

$$
T_{r}=\int_{r_{p}}^{r_{a}} \mathrm{~d} t \frac{u^{t}}{u^{r}}=\int_{0}^{2 \pi} \mathrm{d} \chi \frac{\mathrm{d} r}{\mathrm{~d} \chi} \frac{u^{t}}{u^{r}}
$$

and

$\Delta \phi=\int_{0}^{T_{r}} \mathrm{~d} t \frac{u^{\phi}}{u^{t}}=\int_{0}^{2 \pi} \mathrm{d} \chi \frac{\mathrm{d} r}{\mathrm{~d} \chi} \frac{u^{\phi}}{u^{r}}=\int_{0}^{2 \pi} \mathrm{d} \chi \frac{e p \sin \chi}{(1+e \cos \chi)^{2}} \frac{V_{\phi}(r(\chi))}{\sqrt{V_{r}(r(\chi))}}$.

Results are shown in figures 1,2 and 3 for the pair of orbits $(p, e)=(10 M, 0.5),(15 M, 0.5)$ and $a=0.5 M$. A quantity which nicely illustrates the difference between Kerr and quasi-Kerr orbits is the number of cycles $\mathcal{N}$ required to accumulate $\pi / 2$ difference in periastron shift:

$$
\mathcal{N}=\frac{\pi / 2}{\left|\Delta \phi_{\mathrm{K}}-\Delta \phi_{\mathrm{qK}}\right|}
$$

In figure 3 we show how $\mathcal{N}$ varies with $\epsilon$ and eccentricity $e$ for fixed $p$ and $a$. The dependence on $e$ is quite weak, but there is a strong dependence on $\epsilon$. For example, for a moderate value $|\epsilon|=0.05$ which corresponds to a fractional difference $\sim 8 \%$ in the quadrupole moment, we only need about $\sim 100-200$ orbits to accumulate $\pi / 2$ difference in $\Delta \phi$. Such large orbital dephasing would certainly manifest itself in the waveforms as we shall see in the following section.

\section{Waveform comparisons}

\subsection{Kerr versus quasi-Kerr}

As we already pointed out in section 3.1, the calculation of rigorous gravitational waveforms and fluxes is a major challenge for any non-Kerr EMRI program. For the purposes of this 

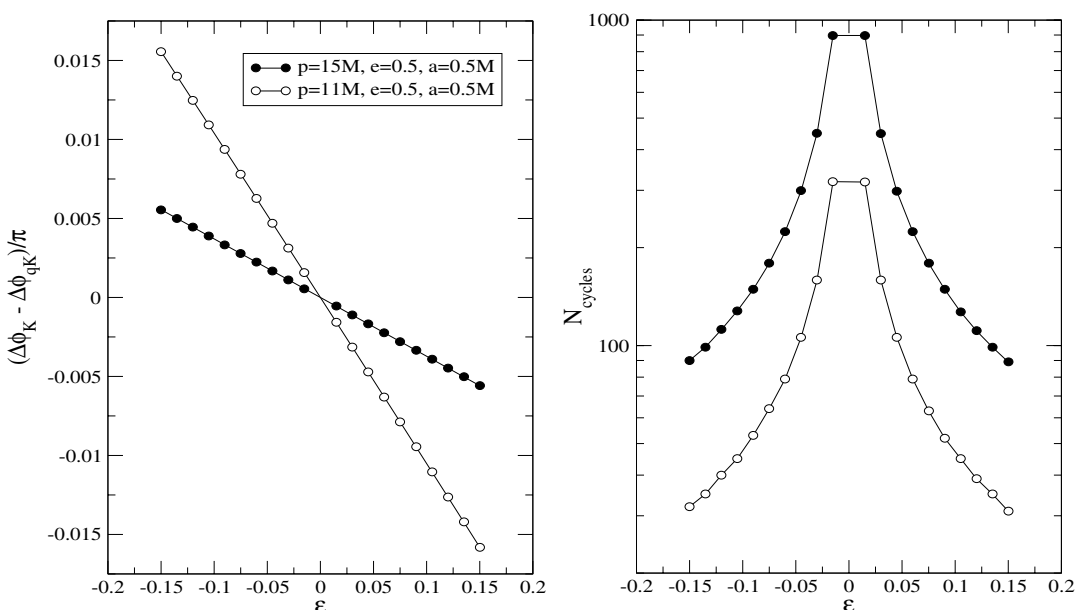

Figure 1. Left panel: periastron shift difference $\Delta \phi_{\mathrm{K}}-\Delta \phi_{\mathrm{qK}}$ as a function of the deviation $\epsilon$. Right panel: number of cycles $\mathcal{N}$ required to accumulate $\pi / 2$ difference in periastron shifts (defined by equation (3.25)) as a function of $\epsilon$. For both panels, we have considered two orbits: $a=0.5 M, e=0.5$ and $p=10 M, p=15 M$.

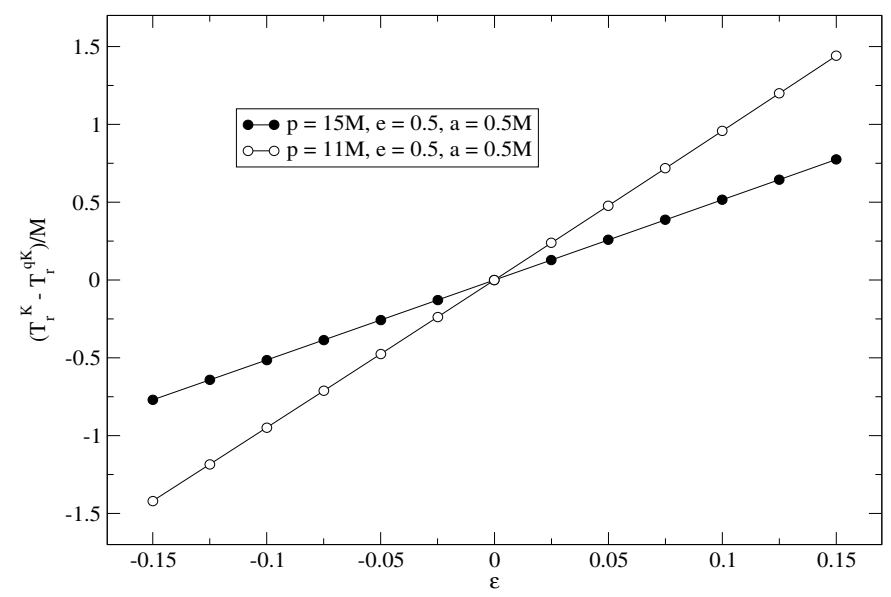

Figure 2. Difference in the radial period $T_{r}$ of Kerr and quasi-Kerr orbits with $a=0.5 M, e=0.5$ and $p=10 M, p=15 M$.

study, which is just an initial investigation, it will suffice to use approximate waveforms, in particular the 'hybrid' waveforms discussed in $[8,24]$. These waveforms are generated using flat spacetime wave formulae (see $[13,31]$ for more details) for the two components $\left\{h_{\times}, h_{+}\right\}$, coupled with exact relativistic geodesic motion. At least for the particular case of Kerr, it has been established that these waveforms agree quite well with rigorous Teukolsky-based waveforms [24]. Hence, it makes sense to assume that the same will be true for the quasi-Kerr case.

In this section we shall compare quasi-Kerr against Kerr waveforms with identical orbital parameters $(p, e)$, spin $a / M$ and for the same kinematic initial conditions. Since a nonzero $\epsilon$ 


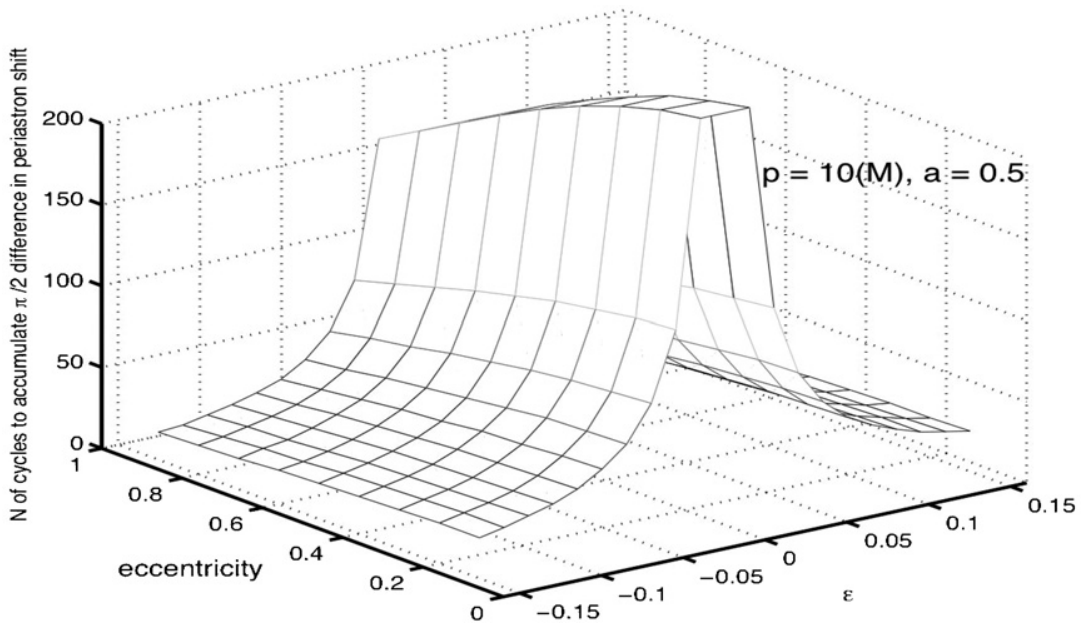

Figure 3. Number of cycles to accumulate $\pi / 2$ difference between Kerr and quasi-Kerr periastron shifts as a function of $\epsilon$ and eccentricity $e$. We have fixed the other two parameters: $p=10 M, a=0.5 M$.

imparts a change in the orbital frequencies we would expect this change to manifest itself as a significant cumulative phase difference between the corresponding waveforms after several orbits. This difference can be rigorously quantified in terms of the overlap function, that is, the scalar product between the normalized waveforms within the LISA sensitivity band:

$$
\left(\hat{h}_{1}, \hat{h}_{2}\right)=4 \Re \int_{0}^{\infty} \frac{\tilde{h}_{1}(f) \tilde{h}_{2}^{*}(f)}{S_{h}(f)} \mathrm{d} f,
$$

where a tilde denotes the Fourier transform of the waveform, a star stands for the complex conjugate, a hat denotes normalized waveform $h\left(t_{k}\right)$ according to $\left(\hat{h}_{1}, \hat{h}_{1}\right)=\left(\hat{h}_{2}, \hat{h}_{2}\right)=1$ and $S_{h}(f)$ is the expected LISA sensitivity function (one-sided power spectral density). To calculate this latter function we use an analytic fit (discussed in [24]) to the numericallygenerated sensitivity curve of [32], for one year of observation and omitting any confusion noise $^{12}$.

The overlap defined in (4.1) is closely related to the signal-to-noise ratio (SNR) [33], which is given by

$$
S N R=(\hat{h}, s)=\mathcal{A}(\hat{h}, \hat{h})=\mathcal{A}=(s, s)^{1 / 2} .
$$

Here we have assumed that the template $\hat{h}$ exactly matches the true signal $s$, and $\mathcal{A}$ is the amplitude of the signal in units of the normalized waveform $\hat{h}$. An imperfect template or a disagreement between the template's parameters and those of the signal results in a reduced SNR,

$$
S N R=\left(\hat{h}_{1}, s\right)=\mathcal{A}\left(\hat{h}_{1}, \hat{h}_{2}\right)<\mathcal{A} .
$$

In this expression we have assumed that the normalized template $\hat{h}_{1}$ does not exactly match the signal $s=\mathcal{A} \hat{h}_{2}$. In other words, a low overlap between a template and the expected signal implies a drop in SNR (which is accompanied by a drop in the event rate due to the decrease of the observable spatial volume).

${ }^{12}$ We have observed that addition of confusion noise does not affect the overlaps, although it slightly affects the estimated dephasing time scale. 


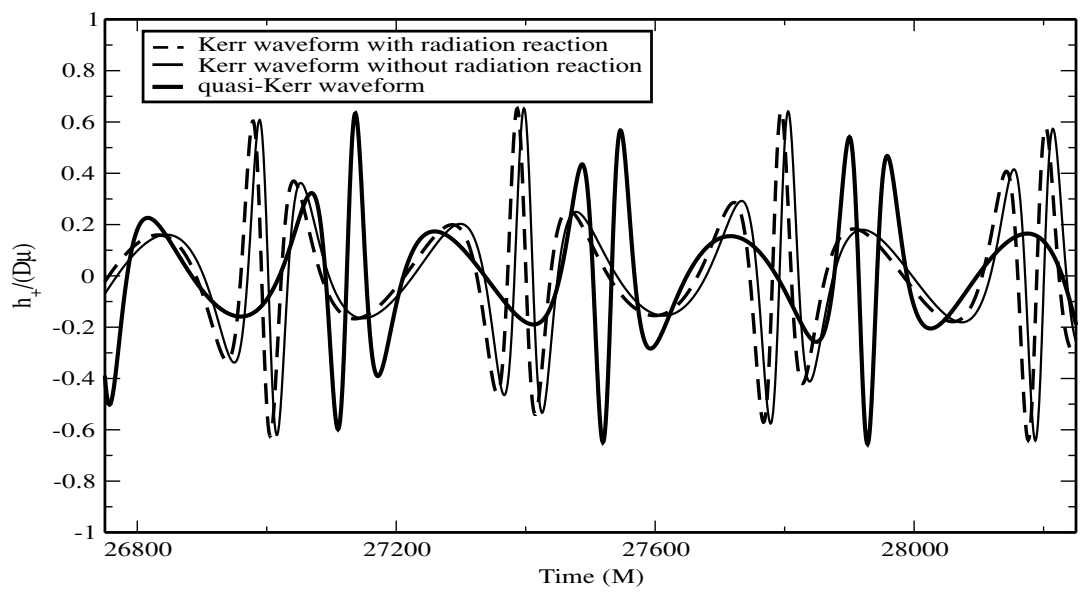

Figure 4. Comparing quasi-Kerr (thick solid curve) and Kerr (thin solid curve) approximate hybrid waveforms for the orbit $p=10 \mathrm{M}, e=0.5, a=0.5 \mathrm{M}$ and for $\epsilon=0.15$. In addition, we include a Kerr waveform taking into account backreaction on the orbit (assuming the same initial orbit), represented by the dashed curve. All waveforms are shown at a time window close to the dephasing timescale $T_{\text {deph }}$.

Since we neglect any radiation reaction effect on the orbital motion our calculations will be consistent provided we 'truncate' any waveform at the radiation reaction time scale $T_{\text {deph }}$. We define $T_{\text {deph }}$ according to the following rule. For a test body in the Kerr spacetime we can include orbital backreaction using the approximate hybrid method $[8,23,34]$. Then comparing Kerr waveforms with and without radiation reaction (denoted as $h_{K}(t), h_{K}^{R R}(t)$, respectively) we can define $T_{\text {deph }}$ as the truncation time at which the overlap between these two waveforms drops below $95 \%$.

$$
\left(\hat{h}_{\mathrm{K}}\left(t=T_{\mathrm{deph}}\right), \hat{h}_{\mathrm{K}}^{\mathrm{RR}}\left(t=T_{\mathrm{deph}}\right)\right)=0.95 .
$$

The particular threshold 0.95 was chosen as it coincides with the usual value of "minimal match' for constructing template banks $[33,35]$. Based on our definition for $T_{\text {deph }}$ we can safely approximate the small body's motion as a geodesic for a time interval $t \lesssim T_{\text {deph }}$. We have numerically computed $T_{\mathrm{deph}}$ (see figure 5) as a function of $\mu / M$, and not surprisingly, we find a dependence, $T_{\text {deph }} \sim M(M / \mu)^{1 / 2}$. This scaling is characteristic of the so-called dephasing timescale, that is, the phase evolution timescale under radiation reaction [36].

For the purpose of waveform comparison we select the moderately relativistic orbit $p=10 M, e=0.5, a=0.5$. For a mass ratio $\mu / M \sim 10^{-5}$ we have $T_{\text {deph }} \approx 27500 M$. The quadrupolar deviation is fixed at $\epsilon=0.15$ which corresponds to a fractional difference $\delta \mathcal{Q} / \mathcal{Q}^{\mathrm{K}}=40 \%$. The actual waveforms are shown in figure 4 . Clearly, after a time lapse $\sim T_{\text {deph }}$ the accumulated phase difference between the two waveforms is quite significant. The waveform deviation due to a nonzero $\epsilon$ can be also compared against the deviation imparted by allowing the orbit to evolve under radiation reaction. For the particular case of figure 4 , after a time interval $t \sim T_{\text {deph }}$, the waveforms dephase mainly due to the non-Kerr property, rather than due to radiation reaction.

In order to quantify the waveform difference in a more rigorous manner we compute overlaps between Kerr and quasi-Kerr waveforms truncated at $t=T_{\text {deph }}$. Some results are given in figure 5 as a function of the mass ratio for the two values $\epsilon=0.07$ and $\epsilon=0.15$. 

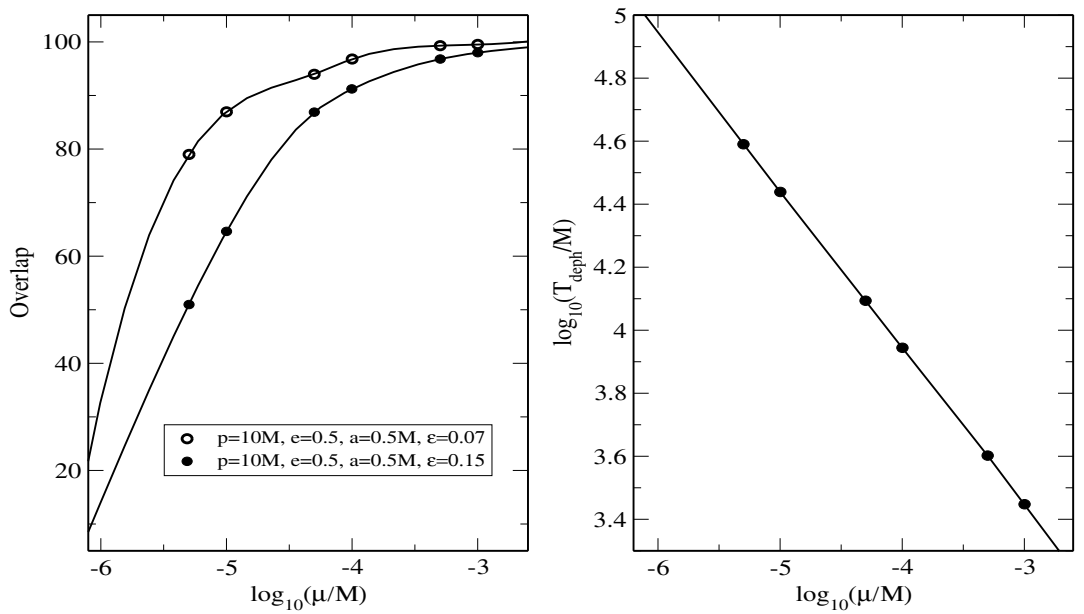

Figure 5. Right panel: radiation reaction timescale $T_{\text {deph }}$ (see the main text for definition), as a function of mass ratio $\mu / M$. Left panel: overlaps (expressed in \%) between quasi-Kerr and Kerr waveforms for the same orbit $p=10 M, e=0.5, a=0.5 M$ and for $\epsilon=0.07,0.15$, truncated at $t=T_{\mathrm{deph}}(\mu / M)$. The solid line is the spline interpolation between the data points.

One can see that the overlap can drop quite dramatically (down to $40 \%$ ) even for small values of $\epsilon(0.07)$ and for realistic mass ratios $\left(\sim 10^{-6}\right)$.

This an important result of the present paper: the typically low overlaps between quasiKerr and Kerr waveforms (for the same orbital parameters and spin) simply mean that using Kerr templates for studying EMRIs in a true non-Kerr spacetime might result in low SNRs, accompanied by significant loss in the number of observed events.

\subsection{Prelude to the 'confusion problem'}

According to our results, it is clear that even a modest (say $\sim 10 \%$ ) deviation from the Kerr quadrupole moment is sufficient to render any Kerr template waveform inaccurate assuming the same orbit $\{p, e\}$ and spin parameter $a / M$.

This statement begs the question: is it actually best to compare waveforms corresponding to the same set of parameters $\{a, p, e\}$ ? One could argue that we should have rather fixed the orbital frequencies,

$$
\Omega_{i}^{\mathrm{K}}=\Omega_{i}^{\mathrm{qK}}, \quad i=\phi, r .
$$

Then one would expect a good agreement in phase between the two waveforms. Note that this choice would correspond in comparing waveforms with different $\{p, e\}$, given fixed values of $a, \epsilon$. If $(p, e)$ and $(\tilde{p}, \tilde{e})$ are the Kerr and quasi-Kerr orbital elements, respectively, and assuming a small deviation $\delta p=\tilde{p}-p \sim \mathcal{O}(\epsilon)$ (and similarly for $e$ ) we have

$$
\Omega_{i}^{\mathrm{K}}(p, e)=\Omega_{i}^{\mathrm{qK}}(\tilde{p}, \tilde{e})=\Omega_{i}^{\mathrm{K}}(\tilde{p}, \tilde{e})+\epsilon \Omega_{i}^{(1)}(p, e) \Rightarrow \frac{\partial \Omega_{i}^{\mathrm{K}}}{\partial p} \delta p+\frac{\partial \Omega_{i}^{\mathrm{K}}}{\partial e} \delta e=-\epsilon \Omega^{(1)}(p, e) .
$$

This $2 \times 2$ system has a non-vanishing determinant and a unique solution for $(\delta p, \delta e)$. Note that for $\epsilon=0$ this solution becomes trivial, but this is true as long as we do not allow for 


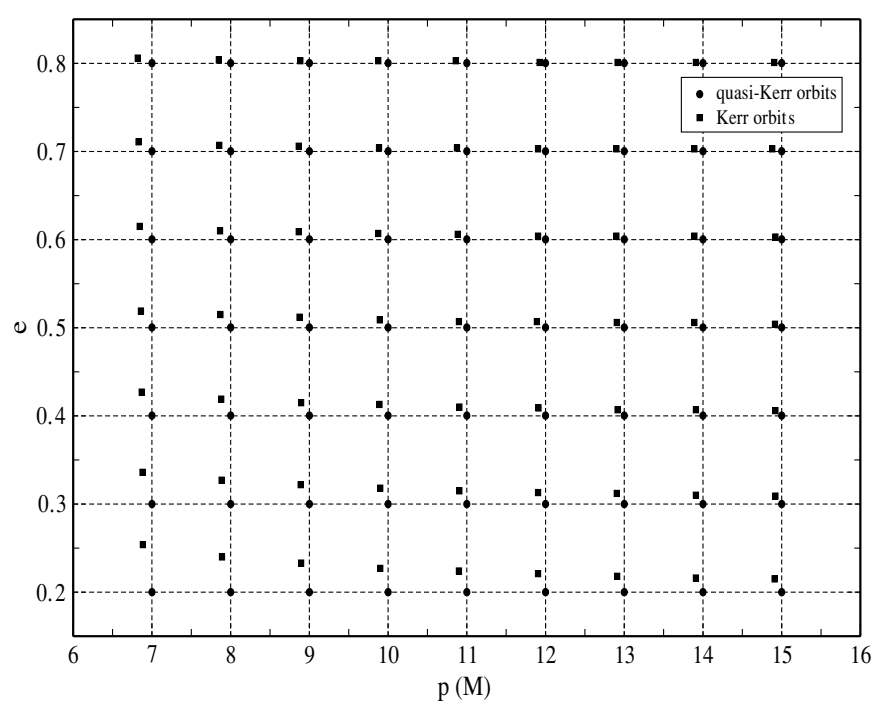

Figure 6. Deviation in the orbital elements $p, e$ between Kerr (squares) and quasi-Kerr (circles), after equating the orbital frequencies $\Omega_{r}, \Omega_{\phi}$, and for fixed $a=0.4 M, \epsilon=0.1$.

variation in $a / M$. In figure 6 we show the solution of (4.5) for $a=0.4 M, \epsilon=0.1$. We can see that the most pronounced deviation between Kerr and quasi-Kerr orbits occurs in the small $p$, small $e$ region. This is understandable as in this case the body spends most of its orbital time in the strong field where the deviation from the Kerr metric is stronger. As expected for $p \gg M$ both $\delta p, \delta e \rightarrow 0$.

Our preliminary results on waveform comparison are quite alarming: fixing the orbital frequencies instead of $\{p, e\}$ lead to overlaps very close to unity between Kerr and quasiKerr waveforms. For the particular case of $\mu / M=10^{-5}, a=0.3 M$ and $\epsilon=0.1$ and for $\{\tilde{p}, \tilde{e}\}=\{10 M, 0.3\}$ we get from (4.5) $\{p, e\}_{K}=\{9.906 M, 0.317\}$ and the resulting overlap is $97.6 \%$ (for the time interval $T_{\mathrm{deph}} \approx 31000 M$ ). Hence, we have naturally stumbled upon a case of the confusion problem: the possibility that for a given non-Kerr EMRI waveform there might be a corresponding Kerr waveform with a different set of parameters which could mimic the former (that is produce an overlap $\geqslant 95 \%$ ) on the dephasing timescale.

Here we have given one example where this scenario is indeed true, but this is not the end of the story. Allowing a time window, longer than $T_{\mathrm{deph}}$, for which radiation reaction effects become important the overlap could significantly reduce as a consequence of different orbital evolution between Kerr and quasi-Kerr. Orbits which are initially 'tuned' to have almost identical orbital frequencies are not expected to preserve this property for $t \gtrsim T_{\text {deph }}$.

The non-trivial issue of waveform confusion is of great importance for LISA and certainly requires further investigation. We will address it in more detail in a following paper.

\section{Concluding discussion}

One of the LISA mission main science goals will be the 'mapping' of the Kerr spacetime. In order to perform this crucial test of general relativity we should be in a position to prepare data analysis tools capable of gauging any possible deviations from the Kerr metric. In this paper we have introduced a practical scheme which could be a strong candidate for this task. 
Our scheme is based on the notion of a 'quasi-Kerr' metric, that is, a metric that deviates only slightly from the known Kerr metric, while still being stationary and axisymmetric. This deviation can first appear in the value of the spacetime's quadrupole moment. We only consider such deviations and ignore any other possible disagreement in higher multipole moments.

Our quasi-Kerr metric is built with the help of the exterior Hartle-Thorne metric, which describes the spacetime outside any slowly-rotating, stationary axisymmetric body. Given this metric, we have first studied the geodesic motion of a test body. Despite the fact that the Hamilton-Jacobi equation is non-separable, we manage to derive approximate equations for generic orbits employing canonical perturbation theory.

In this paper we mostly focused on equatorial orbits (in which case the Hamilton-Jacobi equation is trivially separable) and for given orbital parameters and spin we computed the change imparted to the orbital frequencies and periastron advance by the non-Kerr metric deviation. We found that for (say) a $\sim 10 \%$ fractional difference in the quadrupole moment, the cumulative change in these quantities is significant after about $\sim 100$ orbits, and even for moderately relativistic orbits, it significantly affects the gravitational wave phasing. This was demonstrated by generating approximate 'hybrid' waveforms (for orbits with identical $p, e$ ), for both Kerr and quasi-Kerr spacetimes, and computing their mutual overlap function.

These overlaps typically drop below $\sim 60 \%$ for the relevant mass ratio (allowing for a time interval at which any radiation reaction effects are still not important). Such low overlaps mean that we might expect a significant loss in the signal-to-noise ratio if we attempt to use Kerr waveform templates for the detection of a gravitational wave signal generated by an EMRI in a quasi-Kerr field. This statement holds as long as we compare waveforms with the same orbital elements. However, the picture becomes more blurred when we compare waveforms which correspond to different orbits but same orbital frequencies. Then it is possible for a pair of Kerr and quasi-Kerr waveforms to match extremely well. This confusion problem could be a generic feature of any non-Kerr data analysis effort, and it means that we may confuse a possible true non-Kerr event with a Kerr EMRI with the wrong parameters. A possible way to break this degeneracy is to include backreaction effects, in which case orbits with initial identical frequencies diverge on the radiation-reaction timescale. This issue will be further analysed in a following paper. Similarly, we shall extend the present calculations to the case of non-equatorial orbits, but we expect that the conclusions of the present paper will remain true in this more general scenario.

The scheme we propose here clearly requires further development, especially towards the direction of computing rigorous quasi-Kerr inspiral waveforms. The subject of wave dynamics in a general vacuum axisymmetric-stationary spacetime, or in a less general spacetime like the quasi-Kerr, is almost unexplored. Work in progress aims to investigate if it would be useful to work within the Newman-Penrose formalism [16], trying to formulate some approximate 'quasi-Teukolsky' equation, or if it would be more practical to work directly with metric perturbations.

\section{Acknowledgments}

KG thanks Nils Andersson, Leor Barack, Scott Hughes, John Miller and James Vickers for very fruitful discussions and acknowledges support from PPARC Grant PPA/G/S/2002/00038. SB would like to thank Luc Blanchet for useful comments during the GWDAW9 meeting. We also thank Steve Drasco for his comments on the original version of this paper. 


\section{Appendix A}

Here we provide the explicit form of the various functions appearing in the HT metric, equation (2.6)

$$
\begin{aligned}
F_{1}(r, \theta)= & -\frac{1}{8 M r^{4}(r-2 M)}\left[(r-M)\left(16 M^{5}+8 M^{4} r-10 M^{2} r^{3}-30 M r^{4}+15 r^{5}\right)\right. \\
& +\cos ^{2} \theta\left(48 M^{6}-8 M^{5} r-24 M^{4} r^{2}-30 M^{3} r^{3}\right. \\
& \left.\left.-60 M^{2} r^{4}+135 M r^{5}-45 r^{6}\right)\right]+A_{1}(r, \theta) \\
\equiv & f_{1}(r)+f_{2}(r) \cos ^{2} \theta \\
F_{2}(r, \theta)= & -\frac{5(r-M)\left(2 M^{2}+6 M r-3 r^{2}\right)}{8 M r(r-2 M)}\left\{1-3 \cos ^{2} \theta\right\}-A_{1}(r, \theta) \\
\equiv & f_{3}(r)+f_{4}(r) \cos ^{2} \theta \\
G_{1}(r, \theta)= & \frac{\left(P-72 M^{5} r\right)-3 \cos ^{2} \theta\left(L-56 M^{5} r\right)}{8 M r^{4}(r-2 M)}-A_{1}(r, \theta) \equiv g_{1}(r)+g_{2}(r) \cos ^{2} \theta, \\
H_{1}(r, \theta)= & \frac{\left(16 M^{5}+8 M^{4} r-10 M^{2} r^{3}+15 M r^{4}+15 r^{5}\right)}{8 M r^{4}}\left\{1-3 \cos ^{2} \theta\right\}+A_{2}(r, \theta) \\
\equiv & h_{1}(r)+h_{2}(r) \cos ^{2} \theta, \\
H_{2}(r, \theta)= & \frac{5\left(2 M^{2}-3 M r-3 r^{2}\right)}{8 M r}\left\{1-3 \cos ^{2} \theta\right\}-A_{2}(r, \theta) \equiv h_{3}(r)+h_{4}(r) \cos ^{2} \theta,
\end{aligned}
$$

where

$$
\begin{aligned}
& P(r)=15 r^{6}-45 M r^{5}+20 M^{2} r^{4}+10 M^{3} r^{3}+8 M^{4} r^{2}+80 M^{6} \\
& A_{1}(r, \theta)=\frac{15 r(r-2 M)}{16 M^{2}} \ln \left(\frac{r}{r-2 M}\right)\left\{1-3 \cos ^{2} \theta\right\} \\
& A_{2}(r, \theta)=-\frac{15\left(r^{2}-2 M^{2}\right)}{16 M^{2}} \ln \left(\frac{r}{r-2 M}\right)\left\{1-3 \cos ^{2} \theta\right\}
\end{aligned}
$$

The radial functions $\mathcal{F}_{1,2}(r)$ appearing in the quasi-Kerr metric (2.13) are

$$
\begin{aligned}
& \mathcal{F}_{1}(r)=-\frac{5(r-M)}{8 M r(r-2 M)}\left(2 M^{2}+6 M r-3 r^{2}\right)-\frac{15 r(r-2 M)}{16 M^{2}} \ln \left(\frac{r}{r-2 M}\right) \\
& \mathcal{F}_{2}(r)=\frac{5}{8 M r}\left(2 M^{2}-3 M r-3 r^{2}\right)+\frac{15}{16 M^{2}}\left(r^{2}-2 M^{2}\right) \ln \left(\frac{r}{r-2 M}\right) .
\end{aligned}
$$

\section{Appendix B}

In this appendix we discuss in some detail the Hamilton-Jacoby/action-angle formalism for a test body in the Kerr spacetime, see [13, 30, 37] for full exposition to the topic. Using the definitions and notation of [13] (pp 897-901) we obtain from the Hamilton-Jacobi equation

$$
S_{r}(r)=\int \mathrm{d} r \frac{\sqrt{R}}{\Delta} \quad \text { and } \quad S_{\theta}(\theta)=\int \mathrm{d} \theta \sqrt{\Theta},
$$


where $\Delta=r^{2}-2 M r+a^{2}$. The potentials $R\left(r, \alpha_{k}\right)$ and $\Theta\left(\theta, \alpha_{k}\right)$, where $\alpha_{k} \equiv\left\{-\mu^{2} / 2\right.$, $\left.E, L_{z}, Q\right\}$ the usual constants of motion, are given by well-known expressions, see [13].

The covariant momentum components are $p_{t}=-E, p_{r}= \pm \sqrt{R} / \Delta, p_{\theta}= \pm \sqrt{\Theta}, p_{\phi}=$ $L_{z}$. Incidentally, we point out that an equivalent 'integrated' form of the equations of motion can be written by using $Q^{a}=\partial S / \partial \gamma_{a}$, where we can always make the identification $\gamma_{k}=\alpha_{k}$.

The periodic character of the motion is revealed when we introduce action-angle canonical variables. The actions are defined as (here $i \in\{r, \theta, \phi\}$ ),

$$
J_{i}=\oint p_{i} d q^{i} \Rightarrow\left\{\begin{array}{l}
J_{r}=2 \int_{r_{p}}^{r_{a}} \mathrm{~d} r \sqrt{R} / \Delta \\
J_{\theta}=2 \int_{\theta_{n}}^{\theta_{s}} \mathrm{~d} \theta \sqrt{\Theta} \\
J_{\phi}=2 \pi L_{z}
\end{array}\right.
$$

and are constant functions $J_{i}\left(\alpha_{\kappa}\right)$. In the expression for $J_{\theta}$ we have used $\theta_{n}, \theta_{s}=\pi-\theta_{n}$ for the turning points of the $\theta$-motion.

The next step is to generate the canonical transformation $\left\{x^{\alpha}, p_{\beta}\right\} \Rightarrow\left\{w^{\alpha}, I_{\beta}\right\}$ with $I_{\beta}=$ $\left\{p_{t}, J_{r}, J_{\theta}, J_{\phi}\right\}$. We have the functional forms $I_{\beta}\left(\alpha_{\kappa}\right)$, or $\alpha_{\kappa}\left(I_{\beta}\right)$. Since the new momenta $I_{\beta}$ are constants with respect to $\lambda$ the new Hamiltonian must be $K=K\left(I_{\beta}\right)=K\left(\alpha_{k}\right)$. Hence we can set $K=-\mu^{2} / 2$ and use $W\left(x^{a}, I_{\beta}\right)=S-\mu^{2} \lambda / 2$ as the generating function. For the new coordinates conjugate to $I_{\beta}$ we have

$$
\frac{\mathrm{d} w^{a}}{\mathrm{~d} \lambda}=\frac{\partial K}{\partial I_{\alpha}}=\frac{\partial H_{\circ}}{\partial I_{\alpha}} \equiv v_{\alpha} \Rightarrow w^{a}=v_{a} \lambda+\beta^{a}
$$

with $v_{a}, \beta^{a}$ being constants. As expected, $w^{a}$ are angle variables and $v_{\alpha}$ fundamental frequencies. These frequencies are related to the orbital frequencies (in BL time) as

$$
M \Omega_{i}=v_{i} / v_{t}
$$

The explicit expressions can be found in [30]. We also have

$$
w^{\alpha}=\frac{\partial W}{\partial I_{\alpha}}=\frac{\partial W}{\partial \alpha_{\kappa}} \frac{\partial \alpha_{\kappa}}{\partial I_{\alpha}} .
$$

Expanding equations (B.4),

$$
\begin{aligned}
& w^{t}=v_{t} \lambda+\beta^{t}=-2 v_{t}\left[\frac{\partial S_{r}}{\partial \mu^{2}}+\frac{\partial S_{\theta}}{\partial \mu^{2}}\right]+t-\left[\frac{\partial S_{r}}{\partial E}+\frac{\partial S_{\theta}}{\partial E}\right] \\
& w^{r}=v_{r} \lambda+\beta^{r}=-2 v_{r}\left[\frac{\partial S_{r}}{\partial \mu^{2}}+\frac{\partial S_{\theta}}{\partial \mu^{2}}\right]+\frac{\partial Q}{\partial J_{r}}\left[\frac{\partial S_{r}}{\partial Q}+\frac{\partial S_{\theta}}{\partial Q}\right] \\
& w^{\theta}=v_{\theta} \lambda+\beta^{\theta}=-2 v_{\theta}\left[\frac{\partial S_{r}}{\partial \mu^{2}}+\frac{\partial S_{\theta}}{\partial \mu^{2}}\right]+\frac{\partial Q}{\partial J_{\theta}}\left[\frac{\partial S_{r}}{\partial Q}+\frac{\partial S_{\theta}}{\partial Q}\right] \\
& w^{\phi}=v_{\phi} \lambda+\beta^{\phi}=-2 v_{\phi}\left[\frac{\partial S_{r}}{\partial \mu^{2}}+\frac{\partial S_{\theta}}{\partial \mu^{2}}\right]+\frac{\partial Q}{\partial J_{\phi}}\left[\frac{\partial S_{r}}{\partial Q}+\frac{\partial S_{\theta}}{\partial Q}\right]+\frac{1}{2 \pi}\left[\phi+\frac{\partial S_{r}}{\partial L_{z}}+\frac{\partial S_{\theta}}{\partial L_{z}}\right] .
\end{aligned}
$$

These are the 'integrated' form of the equations of motion and they also provide the $x^{a}\left(w^{k}, I_{\beta}\right)$ relations in an implicit form. From equations (B.5) one can easily derive the usual 'differential' form of the equations of motion; for example, by differentiating the $w^{r}$ and $w^{\theta}$ equations,

$$
\begin{aligned}
& r^{2} R^{-1 / 2} \dot{r}+a^{2} \cos ^{2} \Theta^{-1 / 2} \dot{\theta}+\frac{1}{2} \kappa_{r}\left[\Theta^{-1 / 2} \dot{\theta}-R^{-1 / 2} \dot{r}\right]=1 \\
& r^{2} R^{-1 / 2} \dot{r}+a^{2} \cos ^{2} \theta \Theta^{-1 / 2} \dot{\theta}+\frac{1}{2} \kappa_{\theta}\left[\Theta^{-1 / 2} \dot{\theta}-R^{-1 / 2} \dot{\theta}\right]=1,
\end{aligned}
$$


where here an overdot stands for $\mathrm{d} / \mathrm{d} \lambda$ and

$$
\kappa_{i} \equiv \frac{1}{v_{i}} \frac{\partial Q}{\partial J_{i}} .
$$

Combining equations (B.6),

$\left(\kappa_{r}-\kappa_{\theta}\right)\left[\Theta^{-1 / 2} \dot{\theta}-R^{-1 / 2} \dot{r}\right]=0 \quad \Rightarrow \quad \Theta^{-1 / 2} \dot{\theta}=R^{-1 / 2} \dot{r}=\sigma(r, \theta)$

since $\kappa_{r} \neq \kappa_{\theta}$ for non-equatorial orbits. The remaining function $\sigma(r, \theta)$ is subsequently specified by substitution into either of equations (B.6). We find $\sigma=\left(r^{2}+a^{2} \cos ^{2} \theta\right)^{-1}=\Sigma^{-1}$. Hence, we have arrived at the expected $(r, \theta)$ equations of motion [13]. The remaining $(t, \phi)$ equations can be obtained in a similar fashion from the $\left(w^{t}, w^{\phi}\right)$ equations.

\section{References}

[1] Rees M J 1998 Black Holes and Relativistic Stars: Proc. S. Chandrasekhar Memorial Conference (Chicago: University of Chicago Press) (Preprint astro-ph/9701161)

[2] Menou K, Quataert E and Narayan R 1999 Recent Developments in Theoretical and Experimental General Relativity, Gravitation and Relativistic Field Theories (Singapore: World Scientific)

[3] Colpi M et al 1986 Phys. Rev. Lett. 202485

Mazur P and Mottola E 2004 Proc. Natl Acad. Sci. III 9545

Cea P 2004 Int. J. Mod. Phys. D 131917

Lobo F S N 2006 Class. Quantum Grav. 231525

[4] Danzman K et al 1998 LISA-Laser Interferometer Space Antenna, Pre-Phase A Report Report MPQ 233 Max-Planck-Institute fur Quantenoptic

[5] Thorne K S 1998 Black Holes and Relativistic Stars: Proc. S. Chandrasekhar Memorial Conference (Chicago: University of Chicago Press) (Preprint gr-qc/9706079)

[6] Freitag M and Benz M 2001 Astron. Astrophys. 375711

Freitag M 2001 Class. Quantum Grav. 184033

Freitag M 2003 Class. Quantum Grav. $\mathbf{5 8 3}$ L21

Gair J R, Barack L, Creighton T, Cutler C, Larson S L, Phinney E S and Vallisneri M 2004 Class. Quantum Grav. $21 \mathrm{~S} 1595$

Hopman C and Alexander T 2005 Astrophys. J. 629362

[7] Chakrabarti S K 1996 Phys. Rev. D 532901

Chakrabarti S K 1993 Astrophys. J. 411610

[8] Glampedakis K 2005 Class. Quantum Grav. 22 S605

[9] Mino Y, Sasaki M, Shibata M, Tagoshi H and Tanaka T 1998 Prog. Theor. Phys. Suppl. 128 chapter 1

[10] Ryan F D 1995 Phys. Rev. D 525707

Ryan F D 1997 Phys. Rev D 561845

Ryan F D 1997 Phys. Rev. D 567732

[11] Geroch R 1970 J. Math. Phys. 112580

Hansen R 1974 J. Math. Phys. 1546

Fodor G, Hoenselaers C and Perjés Z 1989 J. Math. Phys. 302252

[12] Sotiriou T P and Apostolatos T A 2005 Phys. Rev. D 71044005

[13] Misner C W, Thorne K S and Wheeler J A 1973 Gravitation (San Francisco: Freeman)

[14] Ryan F D 1997 Phys. Rev. D 556081

[15] Bardeen J M, Press W H and Teukolsky S A 1972 Astrophys. J. 178347

[16] Chandrasekhar S 1983 The Mathematical Theory of Black Holes (Oxford: Oxford University Press)

[17] Teukolsky S A 1973 Astrophys. J. 185635

[18] Stewart J M and Walker M 1974 Proc R. Soc. Lond. A 34149

[19] Carter B 1968 Commun. Math. Phys. 10280

[20] Collins N A and Hughes S A 2004 Phys. Rev. D 69124022

[21] Laarakkers W G and Poisson E 1999 Astrophys. J. 512282

[22] Hartle J B 1967 Astrophys. J. 1501005

Hartle J B and Thorne K S 1968 Astrophys. J. 153807

[23] Glampedakis K, Hughes S A and Kennefick D 2002 Phys. Rev. D 66064005

[24] Babak S V, Fang H, Gair J R, Glampedakis K and Hughes S A 2006 in progress

[25] Abramowicz M A, Almergren G J E, Kluzniak W and Thampan A V 2003 Report Preprint gr-qc/0312070 
[26] Berti E, White F, Maniopoulou A and Bruni M 2005 Mon. Not. R. Astron. Soc. 358923

[27] Glampedakis K and Kennefick D 2002 Phys. Rev. D 66044002

[28] Hughes S A 2000 Phys. Rev. D 61084004

[29] Goldstein H 1950 Classical Mechanics (Reading, MA: Addison-Wesley)

[30] Schmidt W 2002 Class. Quantum Grav. 192743

[31] Press W H 1977 Phys. Rev. D 15965

[32] http://www.srl.caltech.edu/ shane/sensitivity/MakeCurve.html

[33] Cutler C and Flanagan E 1994 Phys. Rev. D 492658

[34] Gair J R and Glampedakis K 2006 Phys. Rev. D 73064037

[35] Balasubramanian R, Sathyaprakash B S and Dhurandhar S 1996 Phys. Rev. D 533033

[36] Detweiler S 2005 Class. Quantum Grav. 22 S681

[37] Carter B 1968 Phys. Rev. 1741559 